\title{
Simulação Computacional do Transporte Horizontal Bidimensional de Contaminantes no Rio Macaé Através da Técnica da Transformada Integral Generalizada
}

\author{
Ademilton Luiz Rodrigues de Souza ${ }^{1}$ \\ Diego Campos Knupp ${ }^{2}$ \\ Pedro Paulo Gomes Watts Rodrigues ${ }^{3}$ \\ Antônio José da Silva Neto ${ }^{4}$ \\ Programa de Pós-Graduação em Modelagem Computacional, Instituto Politécnico, Universidade \\ do Estado do Rio de Janeiro, IPRJ/UERJ, Nova Friburgo, RJ
}

\begin{abstract}
Resumo. Neste trabalho foi desenvolvida uma solução híbrida analítico-numérica através da Técnica da Transformada Integral Generalizada (GITT) empregando-se recursos de programação simbólica através do Software Wolfram Mathematica 10, para solução de um problema bidimensional transiente de dispersão de contaminantes em corpos d' água. Para tal, foi realizada a simulação do comportamento do lançamento instantâneo de um contaminante conservativo $(\mathrm{NaCl})$ em um trecho situado na parte inferior do rio Macaé, localizado no nordeste do Estado do Rio de Janeiro. Para melhor convergência da solução híbrida foram aplicados filtros analíticos para homogeneizar os contornos, e consequentemente, a equação transformada em um sitema acoplado de equações diferenciais parciais unidimensional, resolvido numericamente através de rotinas intrísecas do Mathematica. Os resultados foram comparados com dados experimentais disponíveis, e os resultados obtidos indicam a plausibilidade da solução deste problema através do GITT, o que pode levar a ganhos computacionais, importantes para aplicação em problemas inversos em trabalhos futuros.
\end{abstract}

Palavras-chave. Simulação Ambiental, Contaminantes, GITT, Rio Macaé

\section{Introdução}

Diversos problemas que envolvem fenômenos naturais, físicos, biológicos e sociais podem ser descritos matematicamente por equações diferenciais parciais. Contudo, em grande parte dos casos, a geometria do meio e as condições de contorno inviabilizam a possibilidade de aplicação de técnicas de solução analíticas ou até mesmo de métodos

\footnotetext{
${ }^{1}$ alrsouza@iprj.uerj.br

${ }^{2}$ diegoknupp@iprj.uerj.br

${ }^{3}$ pwatts@iprj.uerj.br

4 ajsneto@iprj.uerj.br
} 
puramente numéricos, pois requerem elevado esforço computacional. Neste contexto, diferentes metodologias surgiram na literatura para tentar combinar a realidade dos métodos analíticos com a flexibilidade dos métodos numéricos. Entre elas destaca-se a técnica da Transformada Integral Generalizada (GITT) que é um método híbrido numérico-analítico, capaz de gerar soluções computacionais bastante eficientes para uma vasta gama de problemas a priori não transformáveis ou não solucionáveis analiticamente [3].

Neste trabalho apresenta-se uma solução analítico-numérica para o caso bidimensional do transporte de contaminantes em regime transiente em um trecho do rio Macaé, Rio de Janeiro. Para o desenvolvimento da solução da equação de advecção-difusão utilizou-se computação simbólica, através da plataforma Wolfram Mathematica.

\section{Descrição do Problema}

A bacia do Rio Macaé é uma das principais bacias hidrográficas do estado do Rio de Janeiro, com área de drenagem de $1.765 \mathrm{~km}^{2}$. O Rio Macaé nasce a 1.560 metros de altitude, próximo ao Pico do Tinguá em Nova Friburgo, no nordeste do estado do Rio de Janeiro. Seu curso percorre cerca de $136 \mathrm{~km}$ de extensão até chegar na cidade de Macaé que deu origem ao seu nome e onde desemboca no Oceano Atlântico [1].

A região de estudo ilustrada pela Figura 1, a seguir, compreende um trecho de $25 \mathrm{~km}$ situado próximo a Usina Termoelétrica Mário Lago localizada no município de Macaé.

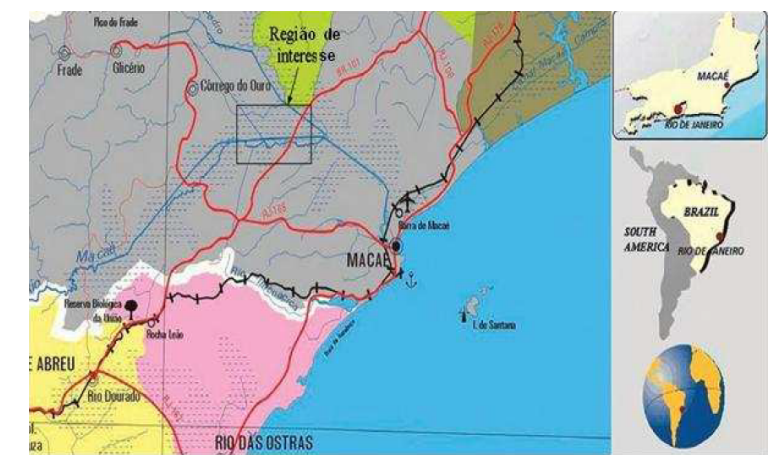

Figura 1: Trecho do Rio Macaé onde está localizada a região de interesse [7].

Para a simulação de uma descarga de poluente no rio Macaé foi utilizado como traçador uma solução de cloreto de sódio $(\mathrm{NaCl})$. Foram utilizados $2.000 \mathrm{~g}$ de $\mathrm{NaCl}$ diluídos em um recipiente contendo 10 litros de água, gerando um volume de aproximadamente 11 litros de solução salina. Esse procedimento foi repetido por 10 vezes, até completar dois recipientes, contendo 55 litros da mistura em cada um. Através de um condutivímetro foi determinada a concentração de $\mathrm{NaCl}$ nos recipientes que foi de $172 \mathrm{mg} / \mathrm{l}$, e ao longo do trecho do rio Macaé que, em condições naturais, foi de $37 \mathrm{mg} / \mathrm{l}$ [10].

Foi traçado um perfil logarítmico de velocidade do rio que, no trecho de estudo, apresentava profundidade média de $1 \mathrm{~m}$ e largura média de $42 \mathrm{~m}$. Para tal, foi utilizado um molinete fluviométrico obtendo os valores de velocidade de $0,5 \mathrm{~m} / \mathrm{s}$ e $0,6 \mathrm{~m} / \mathrm{s}$ a $1 \mathrm{~m}$ e a 
$2 \mathrm{~m}$ da margem, respectivamente. Nas margens a velocidade foi considerada nula. Os dados coletados das concentrações de $\mathrm{NaCl}$ das amostras estão disponíveis em [10], e serão apresentados aqui apenas na forma gráfica, na apresentação dos resultados, na seção 4 .

\section{Formulação e Solução do Problema Direto}

Para a simulação do transporte de contaminantes foi utilizado o modelo bidimensional horizontal uma vez que o trecho de estudo do rio Macaé é classificado como verticalmente bem misturado, ou seja, apresenta homogeneidade ao longo da coluna d'água [1]. Com isso, a formulação matemática desse problema pode ser representada da seguinte forma:

$$
\frac{\partial C(x, y, t)}{\partial t}+U \frac{\partial C(x, y, t)}{\partial x}=E_{L} \frac{\partial^{2} C(x, y, t)}{\partial x^{2}}+E_{T} \frac{\partial^{2} C(x, y, t)}{\partial y^{2}}
$$

onde $0<x<L_{x}, 0<y<L_{y}$, para $t>0$; $U$ é a velocidade do escoamento na direção $x$ e variável na transversal, e $E_{L}$ e $E_{T}$ são os coeficientes de dispersão da concentração longitudinal e transversal respectivamente.

As condições de contorno e inicial são dadas de acordo com as seguintes equações:

$$
\begin{gathered}
C(0, y, t)=C_{0}, 0 \leq y \leq L_{y}, t>0 \\
\frac{\partial C\left(L_{x}, y, t\right)}{\partial x}=0,0 \leq y \leq L_{y}, t>0 \\
\frac{\partial C(x, 0, t)}{\partial y}=\frac{\partial C\left(x, L_{y}, t\right)}{\partial y}, 0 \leq x \leq L_{x}, t>0 \\
C(x, y, 0)=C_{0}+f(x, y), 0 \leq x \leq L_{x}, 0 \leq y \leq L_{y}
\end{gathered}
$$

onde $L_{x}$ e $L_{y}$ são, respectivamente, os comprimentos longitudinal e transversal do trecho em estudo, $C_{0}$ é a concentração normal de $\mathrm{NaCl}$ nas águas do rio Macaé e $f(x, y)=$ $G \delta_{x}\left(x-x_{0}\right) \delta_{y}\left(y-y_{0}\right)$ representa a emissão pontual e instantânea de um poluente na posição $\left(x_{0}, y_{0}\right)$, sendo $G$ a quantidade de massa de poluente emitido.

A solução híbrida analítico-numérica da distribuição de concentração ao longo do trecho, solução das Equações (1) e (2a-d), pode ser obtida utilizando a técnica de transformada integral generalizada (GITT), como descrito na seção seguinte em mais detalhes.

\subsection{Técnica de Transformada Integral Generalizada}

A maioria das técnicas analíticas clássicas são aplicadas para solução de problemas de equações diferenciais relativamente simples. Para problemas mais complexos são utilizados métodos híbridos que envolvem técnicas de soluções analítico-numéricos, como a Técnica da Transformada Integral Generalizada ("Generalized Integral Transform Technique", GITT) [2,3]. Esta técnica consiste na transformação de equações diferenciais parciais em um sistema acoplado e infinito de equações diferenciais ordinárias. O sistema resultante é então numericamente resolvido, após o truncamento em uma ordem $N$, suficientemente grande para atender aos requisitos de precisão da solução. Na sequência, uma fórmula de inversão é usada para obter-se a solução híbrida do problema original. 
Uma das vantagens da GITT é, portanto, a ausência de necessidade de geração de malha espacial, permitindo controle direto do erro global, através da escolha da ordem de truncamento de expansão, $N$. Em geral, para acelerar e otimizar a convergência da técnica GITT, o problema original pode ser simplificado através da proposição de filtros analíticos, de modo a reduzir a importância dos termos-fonte do contorno e equação, se possível tornando-os homogêneos, da seguinte forma:

$$
C(x, y, t)=C_{f}(x)+C^{*}(x, y, t)
$$

onde $C_{f}(x)$ é a solução da função filtro utilizada para homogeneizar as condições de contorno do problema $(1)$, e $C^{*}(x, y, t)$ é a solução do problema homogêneo obtida através da técnica GITT, como uma expansão em autofunções como detalhado a seguir.

\subsection{Problema de Autovalor e Problema Transformado}

A solução do problema original pode também ser encontrada por meio de um esquema de transformação parcial, apenas na direção transversal, resultando em um problema transformado formado por um sistema de equações diferenciais parciais unidimensional.

Seguindo o procedimento para transformada integral, o seguinte problema de autovalor do tipo Sturm-Liouville é proposto:

$$
\begin{gathered}
E_{T} \frac{d^{2} \psi_{i}(y)}{d y^{2}}+\beta_{i}^{2} \psi_{i}(y)=0 \\
\frac{d \psi_{i}(0)}{d y}=0=\frac{d \psi_{i}\left(L_{y}\right)}{d y}
\end{gathered}
$$

A solução do sistema tem suas autofunções e autovalores dados, respectivamente, pelas expressões $\psi_{i}(y)=\cos \frac{x \beta_{i}}{\sqrt{E_{T}}}$ e $\beta_{i}=\frac{\pi i \sqrt{E_{T}}}{L_{y}}$. O problema de autovalor apresentado permite definir o seguinte par de transformação integral, sendo $\widetilde{\psi}_{i}(y)$ as autofunções normalizadas:

$$
\begin{aligned}
& C(x, y, t)=\sum_{i=1}^{\infty} \widetilde{\psi}_{i}(y) \bar{C}_{i}(x, t) \\
& \bar{C}_{i}(x, t)=\int_{0}^{L_{y}} \widetilde{\psi}(y) C(x, y, t) d y
\end{aligned}
$$

Deve ser ressaltado que neste trabalho optou-se por um esquema de transformação parcial, onde somente uma das coordenadas espaciais, onde o efeito difusivo é predominante, é transformada [4], resultando num sistema de equações diferenciais parciais unidimensional a ser resolvido numericamente.

O problema original pode então ser transformado através do operador $\int_{0}^{L_{y}} \widetilde{\psi}(y)-d y$ que é operado em ambos os lados da equação para obter o seguinte problema transformado:

$$
\frac{\partial \overline{C_{i}}(x, t)}{\partial t}+\beta_{i}^{2} \overline{C_{i}}(x, t)-E_{L} \frac{\partial^{2} \overline{C_{i}}(x, t)}{\partial x^{2}}=\sum_{j=1}^{\infty} \frac{d \bar{C}_{j}(x, t)}{d x} \int_{0}^{L_{y}} \widetilde{\psi_{i}} \widetilde{\psi_{j}}(y) U(y) d y
$$


com condições inicial e de contorno transformadas dadas por:

$$
\begin{gathered}
\overline{C_{i}}(x, 0)=\int_{0}^{L_{y}} \widetilde{\psi}_{i}(y) f(x, y) d y \\
\overline{C_{i}}(0, t)=0=\frac{\partial \overline{C_{i}}\left(L_{x}, t\right)}{\partial x}
\end{gathered}
$$

O sistema (6-7) é resolvido numericamente através da rotina NDsolve do Mathematica para os potenciais transformados $\overline{C_{i}}(x, t)$, de modo que a fórmula de inversão, Equação (5a), pode ser empregada para obtenção da solução para o potencial original, $C(x, y, t)$.

\section{Resultados}

Neste trabalho foi considerado um domínio contendo $2.200 \mathrm{~m}$ de comprimento por 40 m de largura. A concentração de $\mathrm{NaCl}$ determinada no contorno em $x=0$ e ao longo do trecho foi de $37 \mathrm{mg} / \mathrm{l}$. No instante inicial foi simulado o lançamento de $20 \mathrm{~kg}$ de $\mathrm{NaCl}(\mathrm{G})$ para previsão do comportamento do perfil de concentração com o tempo ao longo da seção transversal na posição $y=0,5 \mathrm{~m}$ localizado a $50 \mathrm{~m}$ a jusante do ponto de lançamento do poluente em $x=1050 \mathrm{~m}$.

Para o trecho em estudo do rio Macaé foram encontrados os valores $E_{L}=0,25 \mathrm{~m}^{2} / \mathrm{s}$ e $E_{T}=0,006 \mathrm{~m}^{2} / \mathrm{s}$ obtidos pelas fórmulas empíricas $E_{L}=5,93 d u_{*}$ e $E_{T}=0,15 d u_{*}$ estabelecidas, respectivamente, por [5] e [6], sendo $d$ a profundidade média e $u_{*}=\left(g d S_{0}\right)^{0,5}$ a velocidade de atrito do escoamento descrita por [8], onde $g$ é a aceleração da gravidade e $S_{0}$ a declividade do rio. Entretanto, de acordo com [8] os coeficientes de dispersão obtidos por essas equações apresentam uma margem de erro de $\pm 50 \%$. Diante disso, [10] consideraram outras variações desses valores $E_{L}=0,4 \mathrm{~m}^{2} / \mathrm{s}$ e $E_{T}=0,003 \mathrm{~m}^{2} / \mathrm{s}, \mathrm{com} \mathrm{o}$ intuito de se obter uma melhor simulação do perfil de concentração.

A Tabela 1 mostra o comportamento da convergência da solução a medida em que se aumenta a ordem de truncamento $(N)$ do sistema infinito, de modo a atender o nível de precisão da solução e manter o mínimo esforço computacional. Observa-se que os resultados apresentados aparentemente convergiram para, pelo menos, dois dígitos significativos.

Tabela 1: Análise de convergência da solução em relação a ordem de truncamento utilizando a

\begin{tabular}{|c|c|c|c|c|c|c|c|c|c|c|c|}
\hline $\mathrm{x}$ & $\mathrm{N}=20$ & $\mathrm{~N}=40$ & $\mathrm{~N}=60$ & $\mathrm{~N}=80$ & $\mathrm{~N}=100$ & $\mathrm{Y}$ & $\mathrm{N}=20$ & $\mathrm{~N}=40$ & $\mathrm{~N}=60$ & $\mathrm{~N}=80$ & $\mathrm{~N}=100$ \\
\hline \multicolumn{12}{|l|}{$\mathrm{t}=100$} \\
\hline 1100 & 301,9660 & 284,2570 & 287,3910 & 290,2100 & 291,8950 & 0 & 315,986 & 291,55 & 287,968 & 287,709 & 287,97 \\
\hline 1125 & 38,4748 & 38,2170 & 38,2734 & 38,3217 & 38,3158 & 10 & 48,9798 & 46,0267 & 45,6098 & 45,717 & 45,658 \\
\hline 1150 & 37,0004 & 37,0004 & 37,0005 & 37,0004 & 37,0003 & 20 & 31,5606 & 34,0889 & 33,8933 & 33,8515 & 33,8477 \\
\hline 1175 & 37,0000 & 37,0000 & 37,0000 & 37,0000 & 37,0000 & 30 & 37,7054 & 35,8337 & 35,5116 & 35,6077 & 35,5668 \\
\hline 1200 & 37,0000 & 37,0000 & 37,0000 & 37,0000 & 37,0000 & 40 & 39,8533 & 40,013 & 39,6983 & 39,6716 & 39,6769 \\
\hline \multicolumn{12}{|l|}{$t=150$} \\
\hline 1100 & 187,8720 & 215,2270 & 213,1320 & 210,9450 & 209,9220 & 0 & 197,574 & 237,86 & 240,02 & 239,861 & 239,739 \\
\hline 1125 & 201,7760 & 183,6540 & 186,0490 & 187,8350 & 188,9530 & 10 & 39,1082 & 36,9651 & 37,218 & 37,1492 & 37,1817 \\
\hline 1150 & 38,2851 & 38,1835 & 38,2289 & 38,2671 & 38,2556 & 20 & 35,7793 & 36,8251 & 36,9606 & 36,9859 & 36,9953 \\
\hline 1175 & 37,0008 & 37,0010 & 37,0009 & 37,0010 & 37,0010 & 30 & 38,1247 & 36,742 & 36,9564 & 36,8935 & 36,9212 \\
\hline 1200 & 37,0000 & 37,0000 & 37,0000 & 36,9999 & 37,0000 & 40 & 35,4889 & 38,582 & 38,7755 & 38,7914 & 38,791 \\
\hline
\end{tabular}
seção transversal nas posições $y=0,5$ e $x=1100$ a jusante do ponto de lançamento do poluente. 
Nas Figuras 2.a a 2.d, a seguir, observa-se que o comportamento da solução encontrada pelo método GITT é coerente com os dados reais do experimento realizado no rio Macaé. Com as pequenas variações dos coeficientes de difusão verificou-se que as soluções apresentaram uma acentuada sensibilidade do pico de concentração. Além disso, a defasagem observada pode ser atribuída a imprecisão do método de coleta de dados em relação ao campo de velocidades considerado. Observa-se ainda que a curva que mais se adaptou aos dados experimentais foi a da Figura 2.a, onde se utilizou os coeficientes de dispersão calculados empiricamente.

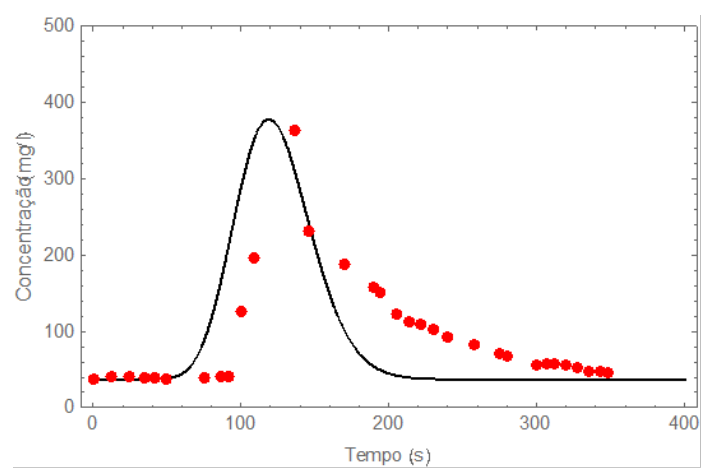

(a) $E_{L}=0,25 \mathrm{~m}^{2} / \mathrm{s}, E_{T}=0,006 \mathrm{~m}^{2} / \mathrm{s}$

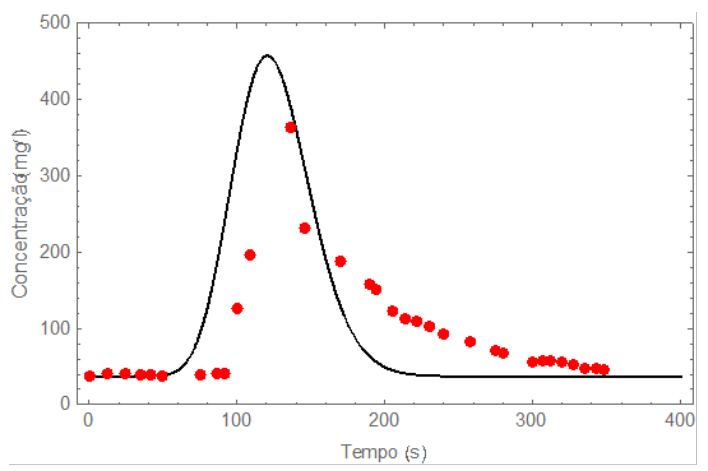

(c) $E_{L}=0,25 m^{2} / s, E_{T}=0,003 m^{2} / s$

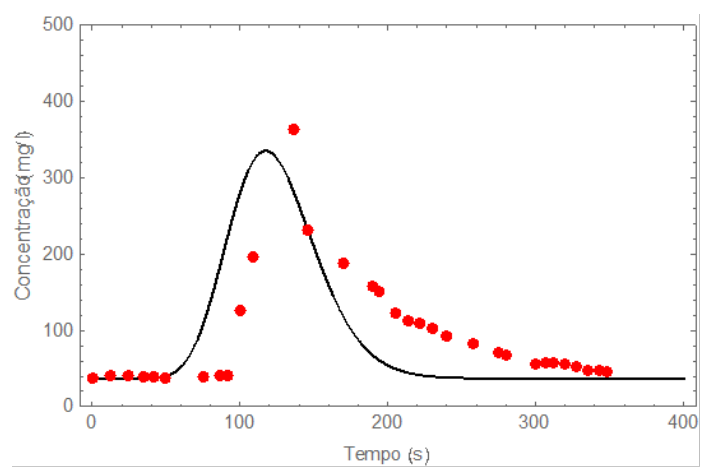

(b) $E_{L}=0,4 m^{2} / s, E_{T}=0,006 m^{2} / s$

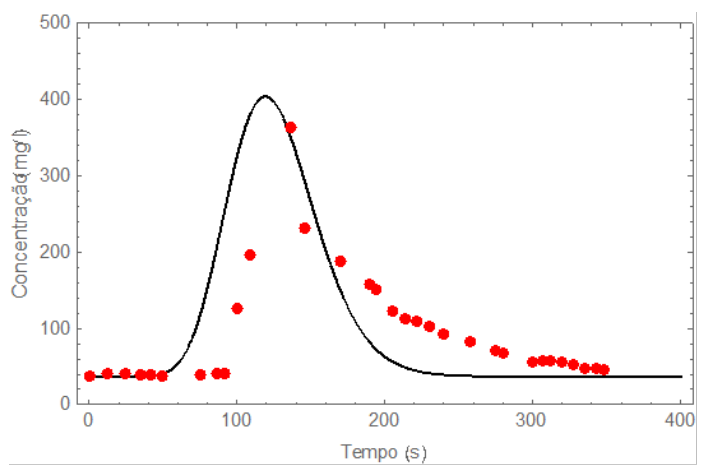

(d) $E_{L}=0,4 m^{2} / \mathrm{s}, E_{T}=0,003 \mathrm{~m}^{2} / \mathrm{s}$

Figura 2: Perfil de concentração de $\mathrm{NaCl}$.

\section{Conclusões}

A análise dos resultados permitiu observar o comportamento da concentração do traçador no corpo hídrico demonstrando que os procedimentos apresentados possibilitam a reprodução de comportamentos físicos esperados, como o decaimento do campo de concentração e deslocamento de seu ponto de máximo em função dos valores dos coeficientes de difusão. Os resultados numéricos demonstraram um comportamento coerente aos obtidos no experimento real. Foi demonstrada a plausibilidade de utilização da GITT na solução deste problema. Esta solução pode ser particularmente interessante para a solução de 
problemas inversos, onde uma solução precisa e rápida computacionalmente é altamente desejável.

Recomenda-se, para trabalhos futuros, a realização de novos experimentos utilizando o método dos problemas inversos para se definir de maneira mais precisa o campo de velocidades (termo advectivo) a fim de reduzir a defasagem encontrada nos resultados.

\section{Agradecimentos}

Os autores agradecem a CAPES, CNPq, FAPERJ e Termomacaé Ltda.

\section{Referências}

[1] K. J. Amaral. Estuário do Rio Macaé: Modelagem Computacional como Ferramenta para o Gerenciamento Integrado de Recursos Hídricos. Dissertação, Universidade Federal do Rio de Janeiro: COPPE, Rio de Janeiro, Brazil, (2003).

[2] R. M. Cotta and M. D. Mikhailov. Heat Conduction: Lumped Analysis, Integral Transforms, Symbolic Computation. Wiley-Interscience, Chichester, UK,(1997).

[3] R. M. Cotta. Integral Transforms in Computational Heat and Fluid Flow. CRC Press, (1993)

[4] R. M. Cotta, D. C. Knupp, C. P. Naveira-Cotta, L. A. Sphaier and J. N. N. Quaresma. The Unified Integral Transforms (UNIT) Algorithm with Total and Partial Transformation. Computational Thermal Sciences, (2014).

[5] J. W. Elder. The dispersion of marked fluid in turbulent shear flow. Journal of Fluid Mechanics, Vol.5, pp. 544-560, (1959).

[6] H. B. Fischer, J. Imberger, E. J. List, R. C. Y. Koh, N. H. Brooks and H. B. Fischer. Mixing in inland and coastal waters. Academic Press, New York, (1979).

[7] J. Lugon Jr., P. P. G. W. Rodrigues and A. J. Silva Neto. Assessment of Dispersion Mechanisms in Rivers by Means of an Inverse Problem Approach. Inverse Problems in Science and Engineering, Vol.16, No.8, pp. 967-979, (2008).

[8] J. L. Martin and S. C. Mccutcheon. Hydrodinamics and Transport for Watter Quality Modeling, Lewis, New York, (1998).

[9] M. N. Özisik. Heat Conduction. 2nd ed., Jonh Wiley \& Sons, (1993).

[10] W. R. Telles, P. P. W. Rodrigues, A. J. Silva Neto, J. L. Junior e M. I. P. Ferreira. Uso de Modelagem Computacional Bidimensional Horizontal com Velocidade Variável na Direção Transversal na Simulação do Transporte de Contaminantes no Rio Macaé, (2009). 